\title{
NOUVELL\&
}

\section{Prévenir la transmission des mutations de I'ADN mitochondrial : mythe ou réalité ?}

Julie Steffann, Sophie Monnot, Agnès Rötig, Arnold Munnich, Jean-Paul Bonnefont

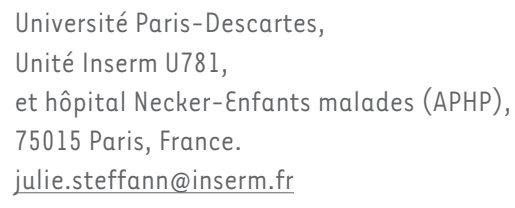

julie.steffann@inserm.fr

> Les mitochondries jouent un rôle central dans le métabolisme énergétique cellulaire. Elles possèdent leur propre génome (ADN mitochondrial, ADNmt), dont la transmission est exclusivement maternelle, et les gènes dédiés à la production de certaines sous-unités des complexes constitutifs de la chaîne respiratoire. Les mutations de l'ADNmt, fréquentes en pathologie humaine, sont responsables de maladies sévères sans traitement curatif à ce jour. Le nombre de copies de l'ADNmt par cellule variant de quelques dizaines à plusieurs centaines de milliers selon le type cellulaire, une mutation de l'ADNmt peut être présente dans toutes les copies d'ADNmt d'un individu (état homoplasmique), ou coexister avec des copies portant la séquence sauvage (état hétéroplasmique) dans des proportions variables, définissant ainsi un taux d'hétéroplasmie. Le taux d'hétéroplasmie est particulièrement important dans la mesure où, au-delà d'un certain «seuil» d'hétéroplasmie, variable d'un tissu à l'autre, d'une mutation à l'autre, et peut-être d'un individu à un autre, apparaît un dysfonctionnement de la chaîne respiratoire. Ce déficit est à l'origine d'une symptomatologie caractérisée par une considérable hétérogénéité intra et interfamiliale pour une mutation donnée. Parmi les pistes thérapeutiques développées dans les maladies mitochondriales, la possibilité de réduire le taux d'hétéroplasmie de l'ovocyte et/ou de l'embryon précoce apparaît donc comme prometteuse.

\section{Stratégies de réduction du taux d'hétéroplasmie mitochondriale}

Compte tenu de la stabilité de la quantité d'ADNmt de la fécondation jusqu'à l'implantation embryonnaire (la réplication mitochondriale est interrompue durant cette période), deux grands types d'approches peuvent théoriquement être utilisés. La première, dénommée «transfert cytoplasmique », consiste à diluer les molécules d'ADNmt mutant en injectant du cytoplasme d'un ovocyte provenant d'une donneuse saine dans l'ovocyte d'une femme porteuse de mutations de l'ADNmt. Réalisée initialement dans le but d'améliorer les méthodes de reproduction assistée [1], cette technique a pour principale limite de nécessiter le transfert d'au moins 30 à $50 \%$ du volume final de l'ovocyte, afin d'obtenir une dilution adéquate de I'ADNmt mutant. Les essais menés chez l'animal font état d'une correction insuffisante (dans tous les cas inférieurs à $25 \%$ [2]) et chez l'homme d'un risque d'anomalie chromosomique [3], voire de modifications épigénétiques [4].

La seconde méthode, nommée «transfert nucléaire »(TN) est plus radicale. Elle consiste à transférer le noyau d'une cellule donneuse porteuse de la mutation de I'ADNmt à éradiquer dans une cellule receveuse saine préalablement énucléée. Suivant le stade où sont prélevés les noyaux, il faut distinguer:

- le transfert de vésicules germinatives entre ovocytes immatures;
- le transfert du fuseau méïotique entre ovocytes matures bloqués en $2^{\mathrm{e}}$ division méiotique ;

- le transfert de pronucléus entre 2 embryons au stade zygote;

- le transfert d'un noyau d'une cellule somatique adulte vers un ovocyte énucléé (technique utilisée pour le clonage), qui n'a pas vraiment d'intérêt pratique dans le cadre de la prévention de la transmission des mutation de I'ADNmt.

Les deux inconvénients majeurs du TN entre ovocytes immatures sont la contamination par des molécules d'ADNmt mutantes du fait de l'accumulation de mitochondries dans l'espace périnucléaire des vésicules germinative d'une part [5], et les faibles capacités développementales des ovocytes maturés in vitro d'autre part [6].

Le TN entre ovocytes bloqués au stade M2 apparaît potentiellement intéressant, en dépit d'une difficulté technique liée à l'absence de délimitation membranaire du complexe synaptonémal. L'utilisation de la microscopie polarisée a récemment permis de surmonter cet obstacle: des essais menés chez l'animal ont prouvé la faisabilité de cette approche, qui a permis la naissance de 3 macaques porteurs de moins de $3 \%$ d'ADNmt du donneur [7]. Reste la contrainte de l'obtention d'ovocytes au même stade de maturation, qui pourrait être contournée par l'application de cette méthode à des ovocytes cryoconservés. 


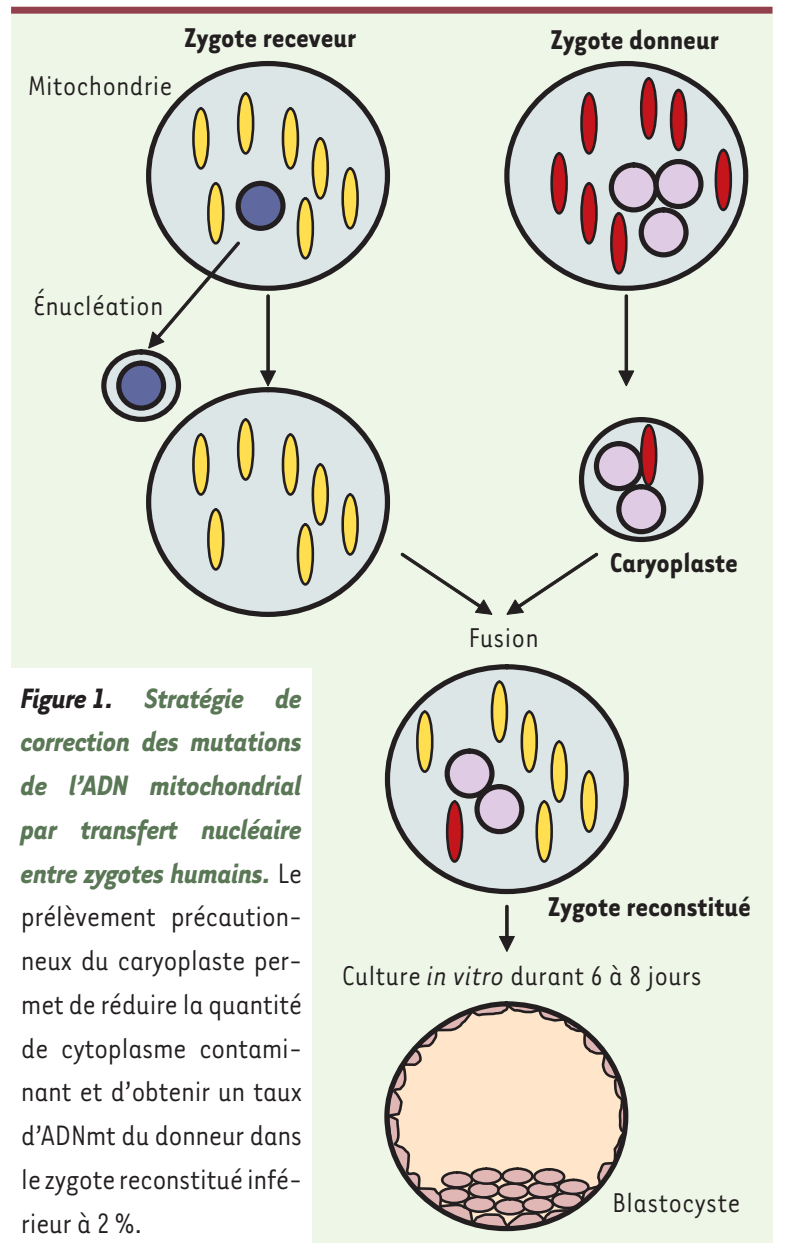

\section{Première application humaine du transfert nucléaire de pronucléus}

L'équipe de D. Turnbull (Newcastle, UK) publie dans Nature [8] la première application humaine du TN de pronuclei entre des embryons au stade zygote résultant d'anomalies de fécondation (mono ou trinucléés). Cette approche avait été préalablement testée dans des modèles murins porteurs d'un polymorphisme de I'ADNmt [9, 10], puis validée chez la souris mitomice portant une délétion de l'ADNmt [11], puisque les souriceaux nés de cette technique étaient indemnes de tout signe clinique et portaient un taux d'hétéroplasmie de l'ordre de $11 \%$. La stratégie consiste à prélever un caryoplaste contenant les pronucléus mâle et femelle du zygote donneur, à le transférer dans la zone pellucide d'un zygote rece- veur préalablement énucléé, à fusionner les membranes grâce à la protéine virale inactivée du virus Sendaï, puis à suivre le développement in vitro des zygotes reconstruits jusqu'au stade de blastocyste (Figure 1). Le taux d'hétéroplasmie mesuré dans les embryons reconstitués a montré une très faible contamination par I'ADNmt du donneur (<2\%). Cependant, cette technique semble influencer négativement le développement embryonnaire puisqu'une perte de $50 \%$ des embryons au stade blastocyste est observée pour les zygotes reconstitués par comparaison aux zygotes porteurs d'anomalies de fécondation mais non micromanipulés.

Ces possibilités de modifications du patrimoine génétique de l'ovocyte ou de l'embryon, qui s'apparentent au clonage, sont de fait interdites dans la plupart des états et des pays, dont la France. II est possible que le législateur n'ait pas pris en compte leur valeur thérapeutique potentielle [12]. Cependant ces approches ne sont pas sans poser un certain nombre de questions, non seulement éthiques [13], mais également scientifiques, en particulier vis-à-vis de leurs éventuelles conséquences sur les interactions noyau-mitochondrie, compte tenu de la provenance tri-parentale des $\mathrm{ADN}^{1}$. Ainsi, le transfert de pronucléus mater-

${ }^{1}$ La provenance triparentale des ADN est la suivante dans le cas du transfert nucléaire: (1) ADN cytoplasmique de I'ovocyte/du zygote receveur; (2) ADN nucléaire pour $1 / 2$ de l'ovocyte «donneur » et pour $1 / 2$ du spermatozoïde. nel entre des embryons de différentes espèces de souris entraîne des anomalies de la morphologie et de la physiologie des souriceaux, probablement par perturbation du dialogue mitochondrie-noyau [14]. De la même façon, l'apparition du phénotype «surdité» chez une lignée de souris mutée dans le gène nucléaire $A h l$ est potentialisée par une mutation du gène mitochondrial codant le tRNA-Arg [15]. Chez l'homme, plusieurs données récentes suggèrent que la pénétrance variable de maladies résultant de mutations de I'ADNmt (neuropathie optique de Leber, surdités non syndromiques, une forme de cardiomyopathie hypertrophique...) est sous la dépendance d'un ou plusieurs gènes nucléaires modificateurs $[16,17]$. Indépendamment de l'implication du génome nucléaire, il est possible que l'état hétéroplasmique soit délétère par lui-même. En 2007, une équipe a ainsi montré que l'induction d'états hétéroplasmiques chez la souris entraînait des effets négatifs sur sa physiologie [18]. Cette question est à mettre en perspective avec la sélection naturelle transgénérationnelle qui s'exerce sur l'ADNmt visant à maintenir ses molécules à l'état homoplasmique (élimination des mitochondries paternelles au moment de la fécondation, phénomène du bottleneck durant l'ovogenèse $[19,20]$ ).

Les détracteurs de ces techniques objectent souvent que des méthodes plus simples de diagnostic prénatal ou préimplantatoire (diagnostic réalisé sur l'embryon conçu in vitro) permettent de dépister précocement ces maladies. Cependant, ces procédures diagnostiques, qui sont fondées sur une mesure du taux d'hétéroplasmie, ne sont pas applicables aux mutations homoplasmiques [21-23]. Par conséquent, en cas de projet parental, les femmes porteuses de mutations homoplasmiques doivent aujourd'hui faire appel à un don d'ovocyte. L'arrivée des procédures de TN offrirait à ces femmes la possibilité de choisir entre le recours 
à un don, procédure dénuée de risque mais contraignante en France (identification d'une donneuse, délai d'attente), ou à un TN dont la sécurité pour l'enfant à naître est loin d'être établie et dont les contraintes seraient identiques. La question au fond qui nous est posée aujourd'hui par ces procédures est de définir la place que nous accordons à la génétique nucléaire dans la parentalité. $\diamond$

Nuclear transfer to prevent

mitochondrial DNA diseases:

myth or reality?

\section{CONFLIT D'INTÉRÊTS}

Les auteurs déclarent n'avoir aucun conflit d'intérêts concernant les données publiées dans cet article.

\section{RÉFÉRENCES}

1. Cohen J, Scott R, Alikani M, et al. Ooplasmic transfer in mature human oocytes. Mol Hum Reprod 1998 ; $4: 269-80$.

2. Ferreira CR, Burgstaller JP, Perecin F, et al. Pronounced segregation of donor mitochondria introduced by bovine ooplasmic transfer to the female germ-line. Biol Reprod 2010 ; 82 : 563-71

3. Barritt J, Willadsen S, Brenner C, Cohen J. Cytoplasmic transfer in assisted reproduction. Hum Reprod Update $2001 ; 7: 428-35$.
4. Hawes SM, Sapienza C, Latham KE. Ooplasmic donation in humans: the potential for epigenic modifications. Hum Reprod 2002 ; 17 : 850-2.

5. Wilding M, Dale B, Marino M, et al. Mitochondrial aggregation patterns and activity in human oocytes and preimplantation embryos. Hum Reprod 2001; $16: 909-17$.

6. Moor RM, Dai Y, Lee C, Fulka J Jr. Oocyte maturation and embryonic failure. Hum Reprod Update 1998; $4: 223-36$.

7. Tachibana M, Sparman M, Sritanaudomchai $H$, et al. Mitochondrial gene replacement in primate offspring and embryonic stem cells. Nature 2009; 461 : 367-72.

8. Craven L, Tuppen HA, Greggains GD, et al. Pronuclear transfer in human embryos to prevent transmission of mitochondrial DNA disease. Nature 2010 ; 465 : 82-5.

9. Meirelles FV, Smith LC. Mitochondrial genotype segregation in a mouse heteroplasmic lineage produced by embryonic karyoplast transplantation. Genetics 1997 ; 145 : 445-51.

10. Meirelles FV, Smith LC. Mitochondrial genotype segregation during preimplantation development in mouse heteroplasmic embryos. Genetics 1998; 148: 877-83.

11. Sato A, Kono T, Nakada K, et al. Gene therapy for progeny of mito-mice carrying pathogenic mtDNA by nuclear transplantation. Proc Natl Acad Sci USA 2005; 102: 16765-70.

12. Roberts RM. Prevention of human mitochondrial (mtDNA) disease by nucleus transplantation into an enucleated donor oocyte. Am J Med Genet 1999; $87: 265-6$.

13. Bredenoord AL, Pennings G, de Wert G. Ooplasmic and nuclear transfer to prevent mitochondrial DNA disorders: conceptual and normative issues. Hum Reprod Update 2008 ; 14 : 669-78.

14. Roemer I, Reik W, Dean W, Klose J. Epigenetic inheritance in the mouse. Curr Biol $1997 ; 7: 277-80$.

15. Johnson KR, Zheng QY, Bykhovskaya Y, et al. A nuclear-mitochondrial DNA interaction affecting hearing impairment in mice. Nat Genet 2001; $27: 191-4$

16. Carelli V, Giordano C, d'Amati G. Pathogenic expression of homoplasmic mtDNA mutations needs a complex nuclear-mitochondrial interaction. Trends Genet 2003; 19: 257-62.

17. Hudson $G$, Keers $S$, Yu Wai Man P, et al. Identification of an X-chromosomal locus and haplotype modulating the phenotype of a mitochondrial DNA disorder. Am J Hum Genet 2005; 77 : 1086-91.

18. Acton BM, Lai I, Shang $X$, Jurisicova A, Casper RF. Neutral mitochondrial heteroplasmy alters physiological function in mice. Biol Reprod 2007; $77:$ 569-76.

19. Cao L, Shitara H, Sugimoto M, et al. New evidence confirms that the mitochondrial bottleneck is generated without reduction of mitochondrial DNA content in early primordial germ cells of mice. PLOS Genet 2009 ; 5 : el 0000756.

20. Sarzi $\varepsilon$, Rötig A. Instabilité du génome mitochondrial et pathologies associées. Med Sci (Paris) 2010 ; $26: 171-6$.

21. Steffann J, Frydman N, Gigarel N, et al. Analysis of mtDNA variant segregation during early human embryonic development: a tool for successful NARP preimplantation diagnosis. J Med Genet 2006 ; $43: 244-7$.

22. Bouchet C, Steffann J, Corcos J, et al. Prenatal diagnosis of myopathy, encephalopathy, lactic acidosis, and stroke-like syndrome: contribution to understanding mitochondrial DNA segregation during human embryofetal development. J Med Genet 2006; 43: 788-92.

23. Steffann J, Gigarel N, Corcos J, et al. Stability of the m.8993T $>$ G mtDNA mutation load during human embryofetal development has implications for the feasibility of prenatal diagnosis in NARP syndrome. J Med Genet 2007 ; 44 : 664-9.

\section{NOUVELL}

Dans la Nouvelle ci-contre $\left(\mathrm{m} / \mathrm{s} \mathrm{n}^{\circ} 10\right.$, vol. 26 , octobre 2010 , page 800 ), un incident technique survenu au niveau des fichiers destinés à l'impression a fait disparaître le nom des 2 auteurs, Amandine Berthet et Erwan Bézard. Nous republions donc ci-contre, les titre, auteurs et coordonnées de cet article.

\section{GRK6, une nouvelle piste thérapeutique pour diminuer les dyskinésies induites par la L-dopa}

Amandine Berthet, Erwan Bézard
Université Victor Segalen-Bordeaux 2, Centre national de la recherche scientifique, Institut des neurosciences de Bordeaux, UMR 5227, 33000 Bordeaux, France. erwan.bezard@u-bordeaux2.fr 